\title{
Patients' role in treatment decision-making
}

\author{
Exploring dental patients' preferred roles in treatment decision-making - a novel approach \\ H. Chapple, S. Shah, A-L. Caress and E. J. Kay Br Dent J 2003; 194: 321-327
}

\section{Aims}

To assess the transferability of the Control Preferences Scale to dental settings and to explore patients' preferred and perceived roles in dental treatment decision-making.

\section{Setting and participants}

A convenience sample of 40 patients, 20 recruited from the University Dental Hospital of Manchester and 20 from a general dental practice in Cheshire.

\section{Methods}

A cross-sectional survey, using the Control Preferences Scale, a set of sort cards outlining five decisional roles (active, semiactive, collaborative, semi-passive, passive), slightly modified for use in dental settings. A second set of cards was used to identify perceived decisional role. Rationale for choice of preferred role was recorded verbatim.

\section{Results}

The Control Preferences Scale was found to be transferable to dental settings. All patients in the sample had identifiable preferences regarding their role in treatment decision-making. A collaborative decisional role, with patient and dentist equally sharing responsibility for decision-making, was most popular at both sites. However, patients at both sites typically perceived themselves as attaining a passive role in treatment decisions. Lack of knowledge about dentistry and trust in the dentist were reported contributors to a passive decisional role preference, whilst those with more active role preferences gave rationales consistent with a consumerist stance.

\section{Conclusions}

This exploratory study's findings suggest that dental patients have distinct preferences in relation to treatment decision-making role and that these may not always be met during consultations with their dentist. The Control Preferences Scale appears to be appropriate for use in dental settings.

\section{IN BRIEF}

- Most dental patients appear to prefer to collaborate with, or even defer to their dentist, rather than take an active role in planning their own treatment.

- Patients who have lost confidence in their dentist seem to have an increased desire for participation in treatment decisions.

- The extent to which patients want to be involved in decisions about their dental treatment depends on many factors, and may vary within an individual, depending on circumstances.

- Identifying and accommodating patients' role preferences had great relevance in dentistry.

\section{COMMENT}

This study introduces the concept of patients' role preferences in relation to dental treatment decision-making; namely the patient having an active, collaborative or passive role within treatment decision-making. It also assesses the transferability of a 'tool' used in medical settings to measure patients' role preference, the Control Preference Scale (CPS), to dentistry.

Forty patients (a convenience sample) were recruited to the study, half from a hospital setting and half from a practice setting. The CPS scale involves the use of a role preference hierarchy using a paired comparisons approach to the content of five sort cards for preferred role, A B C D E. A and B represent active role options, C collaborative options, $D$ and $E$ passive role options. The respondents' perceived role was identified by using a 'pick one' approach of a second set of five sort cards. Differences were observed between preferred and perceived roles in both settings, in that the collaborative role was commonly preferred but a passive role was more commonly perceived as the one attained.

Analysis was undertaken of the preferred data to establish whether an underlying dimension of decisional role preference existed. An underlying 'active-collaborative-passive' dimension was present for the whole group only. Separate analysis for the hospital group and the practitioner group did not show the underlying dimension, possibly due to small numbers.

Supplementary verbatim data were obtained through questions regarding the rationale for choice of most preferred and perceived roles. Five themes emerged: knowledge of subject, trust, consumerist stances, time constraints and factors that would alter individual's role preferences. The factors which altered individual's role preferences included loss of confidence in dentist, increased confidence in the patient, pain and nature of the proposed treatment. Understanding these themes can impact on the development of services, which can empower patients.

The results of this small exploratory study suggest that the CPS is transferable to dentistry. The results of this study also suggest that the relationship between dentist and patient is one of paternalism rather than collaboration.

Partnership between disciplines in healthcare is to be encouraged; here we have an approach normally used in a medical setting applied to dentistry. This type of research is timely when reforming public sector dentistry in the UK is in the spotlight. 1,2 Issues such as consumerism, time constraints and trust in dental practice/practitioners all have an influence on the development of services. Patient-centred care, which empowers individuals towards healthy decisions, is a laudable aim for healthcare workers. Research, which contributes to this aim, can only be welcomed.

Wayne Richards, External Professor of Community General Dental Practice, School of Care Sciences University of Glamorgan. General Dental Practitioner, Swansea.

1. Oliver A. Reforming public sector dentistry in the UK. Br J Health Care Management 2002; 8: 212-216.

2. NHS Dentistry: Options for change, London: $\mathrm{DoH}, 2002$. 